\title{
Growth rate-dependent flexural rigidity of microtubules influences pattern formation in collective motion
}

\author{
Hang Zhou, Naoto Isozaki, Kazuya Fujimoto and Ryuji Yokokawa* (10
}

\begin{abstract}
Background: Microtubules (MTs) are highly dynamic tubular cytoskeleton filaments that are essential for cellular morphology and intracellular transport. In vivo, the flexural rigidity of MTs can be dynamically regulated depending on their intracellular function. In the in vitro reconstructed MT-motor system, flexural rigidity affects MT gliding behaviors and trajectories. Despite the importance of flexural rigidity for both biological functions and in vitro applications, there is no clear interpretation of the regulation of MT flexural rigidity, and the results of many studies are contradictory. These discrepancies impede our understanding of the regulation of MT flexural rigidity, thereby challenging its precise manipulation.

Results: Here, plausible explanations for these discrepancies are provided and a new method to evaluate the MT rigidity is developed. Moreover, a new relationship of the dynamic and mechanic of MTs is revealed that MT flexural rigidity decreases through three phases with the growth rate increases, which offers a method of designing MT flexural rigidity by regulating its growth rate. To test the validity of this method, the gliding performances of MTs with different flexural rigidities polymerized at different growth rates are examined. The growth rate-dependent flexural rigidity of MTs is experimentally found to influence the pattern formation in collective motion using gliding motility assay, which is further validated using machine learning.

Conclusion: Our study establishes a robust quantitative method for measurement and design of MT flexural rigidity to study its influences on MT gliding assays, collective motion, and other biological activities in vitro. The new relationship about the growth rate and rigidity of MTs updates current concepts on the dynamics and mechanics of MTs and provides comparable data for investigating the regulation mechanism of MT rigidity in vivo in the future.
\end{abstract}

Keywords: Microtubule, Flexural rigidity, Localization precision, Growth rate, Collective motion

\section{Introduction}

Microtubule (MT) cytoskeletal filaments play an important role in supporting extended cellular structures, including axons and dendrites, because their flexural rigidity is $2-3$ orders of magnitude higher than that of other cytoskeletal filaments, such as actin and intermediate filaments $[1,2]$. In vivo, the mechanical stiffness of

*Correspondence: yokokawa.ryuji.8c@kyoto-u.ac.jp Department of Micro Engineering, Kyoto University, Kyoto

Daigaku-Katsura, Nishikyo-ku, Kyoto 615-8540, Japan
MTs can be dynamically controlled according to their intracellular function $[3,4]$. In the in vitro reconstructed MT-motor systems, motor-driven MTs can be used as active matter in collective motion or transport of molecular cargoes as nanoscale shuttles or bio-robots [5-10]. As an intrinsic mechanical property of MTs, flexural rigidity determines the fluctuation of the free tip of MTs during the gliding assay and affects MT gliding behavior and orientation [10-14].

Owing to the crucial significance of MT rigidity in biological functions and engineering applications, original author(s) and the source, provide a link to the Creative Commons licence, and indicate if changes were made. The images or other third party material in this article are included in the article's Creative Commons licence, unless indicated otherwise in a credit line to the material. If material is not included in the article's Creative Commons licence and your intended use is not permitted by statutory regulation or exceeds the permitted use, you will need to obtain permission directly from the copyright holder. To view a copy of this licence, visit http://creativecommons.org/licenses/by/4.0/. The Creative Commons Public Domain Dedication waiver (http://creativeco mmons.org/publicdomain/zero/1.0/) applies to the data made available in this article, unless otherwise stated in a credit line to the data. 
numerous measurements on flexural rigidity or persistence length of MTs have been reported using a variety of measurement methods [14-39], such as thermal fluctuations [13, 17, 19, 20, 22, 26, 32], optical tweezers $[18,23,28,38]$, and atomic force microscopy [29]. From these studies, it was found that MT flexural rigidity can be modified by changes in the polymerization conditions, presence of MT-associated proteins (MAPs), and post-translational modification state of the tubulin dimer [40]. However, the reported values of flexural rigidity vary substantially between several studies, as listed in Additional file 1: Table S1. For example, the flexural rigidity of MTs that are polymerized in the presence of guanosine-triphosphate (GTP) and stabilized by paclitaxel, have been reported to vary between 0.03 and $3.2 \times 10^{-23} \mathrm{~N} \cdot \mathrm{m}^{2}$ - two orders of magnitude in range [14-19]. These discrepancies hamper our understanding of the regulatory mechanisms in MT rigidity, thereby challenging the precise manipulation or regulation of MT rigidity and impeding further investigation on the role of flexural rigidity on routine activities, including gliding motility and collective motion.

To address this issue, we developed a new method to evaluate the MT rigidity by improving the conventional measurement methods from two aspects. One is the localization precision of the shape of MTs during image analysis. Most previous studies measured MT flexural rigidity based on the deformation generated by an external force [17-25]. The deformation was measured by skeletonizing the MTs captured in fluorescence or differential interference contrast images and determining their positional shifts. The precision of the skeletonization method varies from 0.02 to 1 pixel, depending on the method. Lower precisions cause a larger measurement error in MT deformation. The other aspect is the variable parameters of polymerization that are not reported in published papers, such as the growth rate of the filaments. As the growth rate is sensitive to experimental conditions such as tubulin concentration, protein source, polymerizing temperature, and the binding of MAPs [41-48], the variation in growth rate causes inconsistency in the measured flexural rigidity.

To demonstrate our method, we investigated the effects of localization precision and growth rate on flexural rigidity. We evaluated MT flexural rigidity at low-level and nanometer-level localization precision using simulation analysis. Further, we modified the growth rate of MTs by altering tubulin concentration and measured flexural rigidity using nanometer-level localization precision. A new relationship between the growth rate and flexural rigidity of MTs was revealed that MT rigidity decreases and goes through three regimes with growth rate increases, which enables us to develop a potential quantitative method for designing the flexural rigidity of MTs.

To test the feasibility of this method, we chose the gliding motility assay as a platform to observe how growth ratedependent flexural rigidity influences the collective motion of MTs, as flexural rigidity has been proven to influence the gliding behaviors and trajectories of MTs [10, 12-14]. Furthermore, we utilized machine learning to recognize, categorize, and verify the distinctive patterns of softer MTs and stiffer MTs.

Our study established a more accurate method of measuring MT rigidity by highlighting the importance of localization precision and growth rate in the measurement process. Furthermore, we developed a standard quantitative method to study the influences of MT rigidity on their routine behaviors by adjusting the growth rate of MTs.

\section{Materials and methods}

\section{Reagent and protein preparations}

All the reagents were purchased from Sigma-Aldrich, unless stated otherwise. Tubulin was purified from porcine brains after two cycles of polymerization and depolymerization [49], resulting in a final tubulin concentration of $282.7 \mu \mathrm{M}$. Recycled tubulin was prepared from tubulin by an additional cycle of polymerization and depolymerization to remove any non-polymerized tubulin. Fluorescently labeled tubulin was prepared by adding $50 \mathrm{M}$ or $15 \mathrm{M}$ excess succinimidyl ester-conjugated tetramethylrhodamine (C-1171, Invitrogen, USA) or Alexa Fluor ${ }^{\mathrm{TM}} 488$ (A-20000, Invitrogen), respectively, to tubulin. His6-tagged kinesin-1 (1 465aa) was purified from Escherichia coli using a nickel-nitrilotriacetic acid affinity resin according to a previously described method [50]. The prepared proteins were stored in liquid nitrogen until use.

\section{MT fluctuation model}

We developed a model using our previous method to simulate the thermal fluctuations of MTs with specific length $(L)$ and defined flexural rigidity $\left(\kappa_{s e t}\right)$ [10]. MTs that were assumed to be tethered at one end behaved as a cantilever beam in this model. The immobilized end was set as the origin of the orthogonal coordinate system. The shape of the MT-free segment, $y(s)$, was expressed by the superposition of sine, cosine, hyperbolic sine, and hyperbolic cosine waves, $W_{n}(s / L)[51]$.

$$
y(s)=\sum_{n=1}^{\infty} \sqrt{\frac{1}{L}} a_{n} W_{n}\left(\frac{s}{L}\right)
$$

and 


$$
\begin{aligned}
W_{n}\left(\frac{s}{L}\right)= & \frac{-\cosh \left(q_{n}\right)-\cos \left(q_{n}\right)}{\sin \left(q_{n}\right)+\sinh \left(q_{n}\right)}\left(\sin \left(\frac{q_{n} s}{L}\right)-\sinh \left(\frac{q_{n} s}{L}\right)\right) \\
& +\cos \left(\frac{q_{n} s}{L}\right)-\cosh \left(\frac{q_{n} s}{L}\right)
\end{aligned}
$$

where, $s$ is the path length from the tethered end along the MT, and $q_{n}$ is $1.875(n=1), 4.695(n=2), 7.855$ $(n=3)$, and $(n-0.5) \pi(n \geq 4)[51]$.

Equating thermal energy with MT bending energy calculated from MT deformation enables us to derive MT flexural rigidity, $\kappa$, with the variance of amplitude in each $n$th mode, $\operatorname{var}\left(a_{n}\right)[52]$.

$$
\kappa=\frac{k_{B} T}{\operatorname{var}\left(a_{n}\right)}\left(\frac{L}{q_{n}}\right)^{4}
$$

Here, $k_{\mathrm{B}}$ is Boltzmann's constant, $T$ is the absolute temperature under experimental conditions (298 K), and $a_{n}$ is the $n$th mode amplitude, which follows a Gaussian distribution with a mean of zero and variance of $\operatorname{var}\left(a_{n}\right)$. The thermal fluctuation model of MTs was generated by setting $L$ to 5,10 , and $30 \mu \mathrm{m}$, and $\kappa_{\text {set }}$ to $0.03,0.3$, and $3 \times 10^{-23} \mathrm{~N} \mathrm{~m}^{2}$, within the range of previously measured rigidities for GTP-polymerized, paclitaxel-stabilized MTs [14-19].

The measured flexural rigidity, $\kappa_{\text {meas }}$, was calculated from the rounded coordinates at different localization precisions. First, the $x y$-coordinates of the shape of MTs in the model were rounded to the nearest multiple of the given localization precision: $1 \mathrm{~nm}(\approx 0.01$ pixels), $10 \mathrm{~nm}$ $(\approx 0.1$ pixels $)$, and $100 \mathrm{~nm}(\approx 1$ pixel $)$. For example, $y=236.8 \mathrm{~nm}$ was converted to $y=237 \mathrm{~nm}$ under a localization precision of $1 \mathrm{~nm}, y=240 \mathrm{~nm}$ under $10 \mathrm{~nm}$, and $y=200 \mathrm{~nm}$ under $100 \mathrm{~nm}$. Thereafter, MT was divided into $N+1$ points with the converted coordinates of $\left(x^{\prime}{ }_{k}\right.$, $\left.y^{\prime}{ }_{k}\right)$. The $n^{\text {th }}$ mode amplitude of the converted shape, $a^{\prime}{ }_{n}$, was Fourier inverse transformed from Eq. (1) and solved using the midpoint method as follows:

$$
a_{n}^{\prime} \approx \sqrt{\frac{1}{L^{\prime}}} \sum_{k=1}^{N} y_{k}^{\prime} \Delta s_{k}^{\prime} W_{n}\left(\frac{s_{k}^{\prime \text { mid }}}{L^{\prime}}\right), n=1, \ldots, N-1
$$

where, $\Delta s^{\prime}{ }_{k}$ is the length of the infinitesimal segment calculated by

$$
\begin{aligned}
& \Delta s_{k}^{\prime}=\sqrt{\left(x_{k+1}^{\prime}-x_{k}^{\prime}\right)^{2}+\left(y_{k+1}^{\prime}-y_{k}^{\prime}\right)^{2}} \\
& L^{\prime}=\sum_{k=1}^{N} \Delta s_{k}^{\prime}
\end{aligned}
$$

and

$$
s_{k}^{\prime \text { mid }}=\Delta s_{1}^{\prime}+\Delta s_{2}^{\prime}+\Delta s_{3}^{\prime}+\cdots+\Delta s_{k-1}^{\prime}+\frac{1}{2} \Delta s_{k}^{\prime}
$$

The $\kappa_{\text {meas }}$ was measured under each localization precision by substituting $a_{n}^{\prime}$ and $L^{\prime}$ into Eq. (3).

\section{Measurement of flexural rigidity}

Seed MTs were polymerized by incubating Alexa Fluor $^{\mathrm{TM}}$ 488-labeled tubulin and recycled tubulin at a 1:2 molar ratio in the presence of $1 \mathrm{mM}$ DTT and $1 \mathrm{mM}$ GMPCPP at $37^{\circ} \mathrm{C}$ for $30 \mathrm{~min}$. The seed MTs were biotinylated by incubating with $20 \mathrm{M}$ excess succinimidyl ester-conjugated biotin (B1606, Invitrogen) at $37{ }^{\circ} \mathrm{C}$ for $30 \mathrm{~min}$ and quenched with $200 \mathrm{M}$ excess potassium glutamate at $37^{\circ} \mathrm{C}$ for $10 \mathrm{~min}$. The partially biotinylated MTs were prepared by incubating the biotinylated seeds in the tubulin solution at various concentrations $(20,30,35,40,50,75,100,150$, and $200 \mu \mathrm{M})$. All MTs were stabilized with $20 \mu \mathrm{M}$ paclitaxel after elongation.

Flow chambers were constructed by bonding the Au-stripe-patterned $\mathrm{SiO}_{2}$ substrate (see Additional file 1: Figure S1 for fabrication details) and a coverslip (C218181, Matsunami Glass, Japan) with $10-\mu \mathrm{m}$ thick double-sided tapes $(7070 \mathrm{~W}$, Teraoka Seisakusho, Japan). We introduced $2 \mathrm{mg} \mathrm{mL} \mathrm{mL}^{-1}$ streptavidin and incubated thrice for 3 min for non-specific binding on the Au-patterned substrates. After washing the flow chamber with BRB80 (80 mM PIPES, 1 mM EGTA, and $1 \mathrm{mM} \mathrm{MgCl}_{2}$ ), the partially biotinylated MTs were immobilized via biotin-streptavidin binding by incubation for $5 \mathrm{~min}$ (Fig. 1A). Free MTs were washed with BRB80 containing an $\mathrm{O}_{2}$ scavenger system $\left(8.0 \mu \mathrm{g} \mathrm{mL} \mathrm{m}^{-1}\right.$ catalase, $25 \mathrm{mM}$ D-glucose, $20 \mu \mathrm{g} \mathrm{mL}^{-1}$ glucose oxidase, $1 \% \beta$-mercaptoethanol, $20 \mathrm{mM}$ DTT, and $20 \mu \mathrm{M}$ paclitaxel). The flow chamber was then sealed with clear nail polish to prevent drying or leakage.

MTs were observed under an IX73 inverted epifluorescence microscope (Olympus, Japan) with an excitation filter (GFP/DsRed-A-OMF, Opto-Line International, Inc., USA), a complementary metaloxide-semiconductor camera (ORCA-Flash 4.0 V2, Hamamatsu Photonics, Japan), image-splitting optics (W-VIEW GEMINI, Hamamatsu Photonics) with a bandpass emitter (FF01-512/25-25 and FF01-630/92-25, Semrock, USA), dichroic mirror (FF560-FDi01-25 × 36, Semrock), and oil-immersion objectives. The magnification and exposure time were set to $100 \times($ NA 1.4) and $100 \mathrm{~ms}$, respectively. To observe MT thermal fluctuation, the frame rate and recording period were set to 2.5 frames $\mathrm{s}^{-1}$ and $200 \mathrm{~s}$, respectively. We used an ND25 filter equipped with a shutter (VMM-D3, Uniblitz, USA). Optical images were stored as sequential image files in TIFF format using the HCImage software 

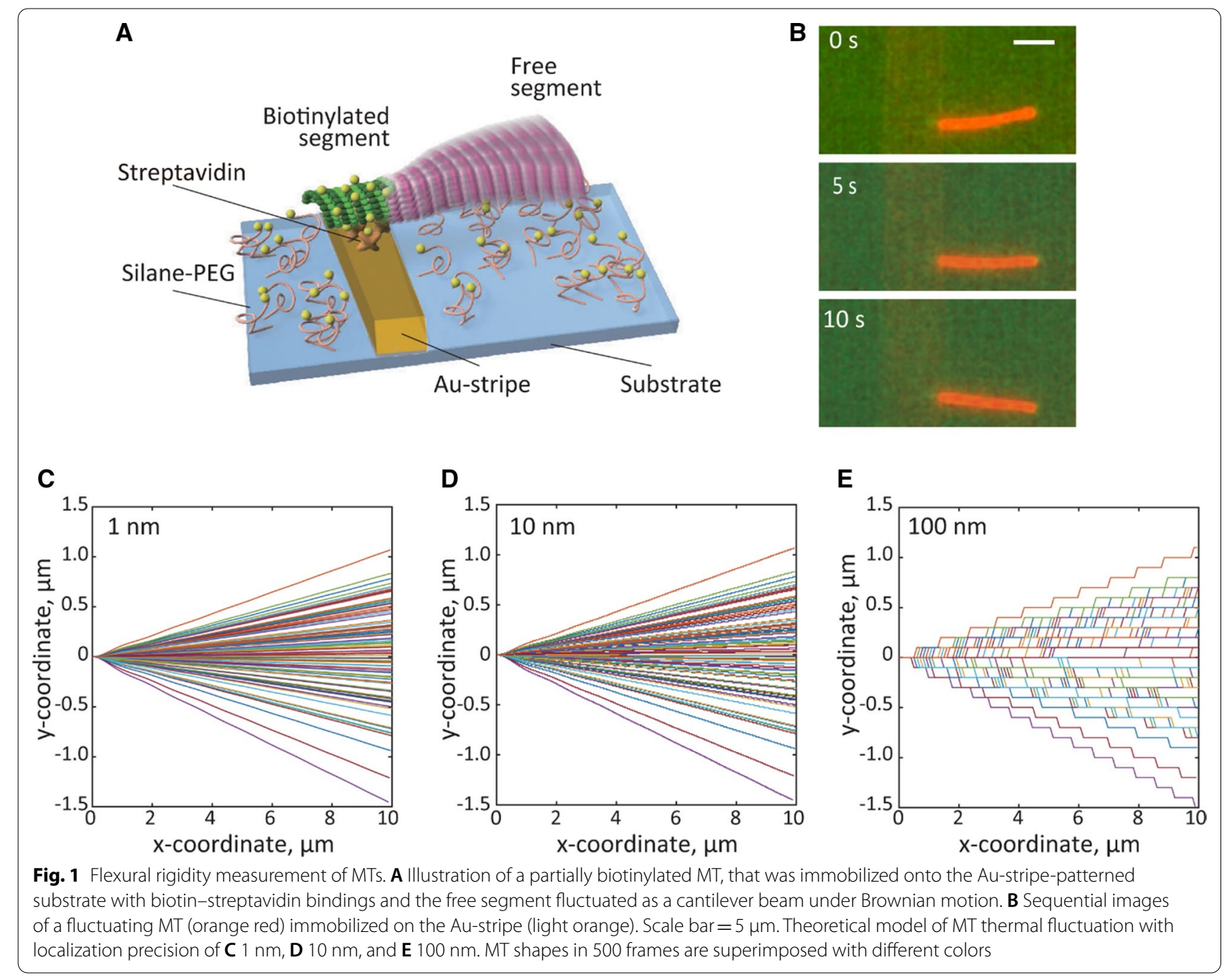

(Hamamatsu Photonics) (Fig. 1B and Additional file 2: Movie S1, Additional file 3: Movie S2, Additional file 4: Movie S3, Additional file 5: Movie S4). The MT shapes were determined using the MT tracking software, fluorescence image evaluation software for tracking and analysis (FIESTA) [53] with sub-pixel resolution (0.02 pixels, $\sim 2.2 \mathrm{~nm}$ ) via $2 \mathrm{D}$ Gaussian fitting (Additional file 1: Figure S2).

Multiple significance tests were performed on all data using Steel-Dwass tests at a critical value of $p<0.05$, and normality or log-normality was tested among outlierremoved data using Lilliefors tests at a critical value of $p>0.05$. All curve fittings were performed using the leastsquares method in MATLAB (R2019b).

\section{Growth rate measurement using TIRF microscopy}

A flow chamber was constructed by bonding two coverslips (C218181 and C024361, Matsunami Glass) with $50-\mu \mathrm{m}$ thick double-sided tapes (400P50, Kyodo Giken Chemical, Japan). First, $50 \mu \mathrm{g} \mathrm{mL} \mathrm{m}^{-1}$ neutravidin was introduced and incubated for $5 \mathrm{~min}$ at $25^{\circ} \mathrm{C}$. After washing the channel thoroughly with BRB80, 1\% Pluronic solution was introduced into the flow cell for $5 \mathrm{~min}$ at $25^{\circ} \mathrm{C}$ and washed twice with BRB80.

The partially biotinylated seed MTs were introduced and selectively immobilized onto substrates via biotinneutravidin binding with a 5 min incubation period. After washing the free seed MTs, a mixture of non-labeled and tetramethylrhodamine-labeled tubulin, $\mathrm{MgSO}_{4}$, and GTP was introduced into the flow cell. The final tubulin concentration was modified to $20,30,35,40,50,75,100$, 150 , and $200 \mu \mathrm{M}$, similar to the flexural rigidity measurements. The flow cell was sealed with clear nail polish and placed on a $37^{\circ} \mathrm{C}$ heater installed on the stage of the microscope.

An EMCCD camera (iXon Ultra 897, Andor Technology, UK) and an inverted microscope (IX 71, Olympus) 
were used for fluorescence observation of the MT elongation process. Laser light sources (DPGL-2050F, Suwtech, China) with a wavelength of $532 \mathrm{~nm}$ were used as the excitation light source. The laser beam path was adjusted such that the excitation light was in the total reflection state. A fluorescent mirror unit (U-MWIG 3, Olympus) was used to separate the excitation light and fluorescence. To measure the elongation rate, 500 fluorescence images were captured with an exposure time of $100 \mathrm{~ms}$, binning $1 \times 1$, using a $60 \times$ objective lens $(\mathrm{APON}$ 60 XOTIRF, Olympus) for total internal reflection fluorescence (TIRF) microscopy. Image data were acquired in the TIFF format using the image acquisition software (Andor iQ 3, Andor). From the obtained sequential fluorescence images, the elongated lengths of the MTs were measured and the elongation rates were calculated using ImageJ (National Institutes of Health). Multiple significance tests were performed on all data using SteelDwass tests with a critical value of $p<0.05$.

\section{In vitro gliding assay}

The coverslips (C218181 and C024361, Matsunami Glass) used for the gliding assay were treated with piranha solution at $60{ }^{\circ} \mathrm{C}$ for $10 \mathrm{~min}$, rinsed with deionized water and then dried with nitrogen gas. The central area $(5 \times 5 \mathrm{~mm})$ of the coverslip was exposed to plasma with the surrounding area covered by tape. Two PIPES-based buffer solutions were used in the assay: (i) casein buffer, with $0.3 \mathrm{mg} \mathrm{mL}^{-1}$ casein dissolved in the BRB80 and (ii) motility buffer, BRB80 with $5 \mathrm{mM}$ ATP, $0.3 \mathrm{wt} \%$ methylcellulose, and $\mathrm{O}_{2}$ scavenger system.

The gliding assay was conducted by introducing MTs onto kinesin molecules with motility buffer (Fig. 5A). First, $2 \mathrm{mg} \mathrm{mL}^{-1}$ streptavidin was introduced and incubated for non-specific binding to the substrate. After washing the chamber with BRB80 solution, biotinconjugated kinesin-1 $\left(0.154 \mathrm{mg} \mathrm{mL}^{-1}\right)$ was added and incubated for $5 \mathrm{~min}$ and immobilized on the glass via biotin-avidin binding. After washing with casein buffer, MTs and motility buffer were introduced into the chamber. All gliding assays were performed at $25{ }^{\circ} \mathrm{C}$. The gliding motion assays were observed using an inverted epifluorescence microscope (IX73, Olympus) with an excitation filter (GFP/DsRed-A-OMF, Opto-Line International, Inc.) and $60 \times$ oil-immersion objectives. The exposure time was set at $50 \mathrm{~ms}$ with a frame rate of 1 frame $\mathrm{s}^{-1}$ and a recording period of $300 \mathrm{~s}$. The addition time of the motility buffer was set as $0 \mathrm{~s}$, and the whole observation time for each assay was $60 \mathrm{~min}$. The captured images were stored as sequential image files in TIFF format using the HCImage software (Hamamatsu Photonics).

\section{MT pattern analysis using machine learning}

A deep convolutional neural network $(\mathrm{CNN})$ model including pre-trained ResNet50 top layers, a global average pooling layer, and a final dense layer activated using the Softmax function was constructed to classify the two MTs (Fig. 5B) [54]. We captured $1400 \mathrm{MT}$ pattern images and 700 images each from softer-MT and stiffer-MT groups from 10 independent experiments for the dataset (840 images for the training process, 360 images for the validation process, and 200 images for the test process). All the images were cropped and resized to $224 \times 224$ pixels as input images for the model. The ground truth of the images was manually labeled. The model was trained by monitoring the validation loss, and the parameters were updated using the Adam optimizer. The model was trained for 20 epochs with a batch size of 32, and a final validation accuracy of $98 \%$ was obtained (Additional file 1: Figure S5). The calculated probabilities for each class were used for MT classification, and the sum of the probabilities was $100 \%$. Tensorflow2.0 (www.tensorflow. org) and Keras API (keras.io) were used during the entire process.

A score class activation map (Score-CAM) was introduced to visually interpret the prediction strategy of the $\mathrm{CNN}$ classifier by obtaining the weights of each activation map on the predicted class [55]. For the deep learning model $f($ ), we used input $X$ and output $Y: Y=f(X)$.

The activation of the input $X_{0}$ generated in the convolution layer $l$ is indicated as $A_{l}$, and the $k$ th channel activation of $A_{l}$ is $A_{l}^{k}$. Further, $A_{l}^{k}$ is extracted, normalized, and upsampled to an input size of $M_{l}^{k} \cdot M_{l}^{k}$ was used as a mask to be projected onto the original image $X_{0}$. For a given baseline $X_{b}$, the contribution $S_{k}^{c}$ of $A_{l}^{k}$ to the output class $c$ is calculated as follows [55]:

$$
S_{k}^{c}=f^{c}\left(M_{l}^{k} \circ X_{0}\right)-f^{c}\left(X_{b}\right)
$$

where, $\circ$ indicates the element-wise product and $\alpha_{k}^{c}$ is the corresponding weight of $A_{l}^{k}$ to the target class $c$.

$$
\alpha_{k}^{c}=\exp \left(S_{k}^{c}\right) / \sum_{k} \exp \left(S_{k}^{c}\right)
$$

The heatmap $L_{\text {score-CAM }}^{c}$ is generated by the linear combination of activation maps $A_{l}^{k}$ and their weights $\alpha_{k}^{c}$ on target class $c$.

$$
L_{\text {score-CAM }}^{c}=\operatorname{ReLU}\left(\sum_{k} \alpha_{k}^{c} A_{l}^{k}\right)
$$

Here, the activation function $R e L U$ was used to select the features with positive importance scores. 


\section{Results and discussion}

\section{Flexural rigidity of MTs under different localization} precisions

The flexural rigidity of MTs was measured from the rounded coordinates at different localization precisions (Fig. 1). Figure 1C-E show the converted shapes of MTs under the localization precision of 1,10 , and $100 \mathrm{~nm}$ with MT length $L=10 \mu \mathrm{m}$ and defined flexural rigidity $\kappa_{\text {set }}=0.3 \times 10^{-23} \mathrm{~N} \mathrm{~m}^{2}$. The shapes in 500 frames were superimposed with different colors. Under low localization precision, the rounded MT shapes were discontinuous and substantially deviated from the original shapes.

The shape and approximate value of $\kappa$ for an MT of $L=10 \mu \mathrm{m}$ and $\kappa_{\text {set }}=0.3 \times 10^{-23} \mathrm{~N} \mathrm{~m}^{2}$ was calculated using Eq. (1), as shown in Fig. 2A, B. The shapes of MTs are represented by the blue line, whereas the fitted curves are indicated by the red lines and red dots with localization precision of (A) $1 \mathrm{~nm}$ and (B) $100 \mathrm{~nm}$. As the localization precision decreases, the thermal fluctuation of MTs cannot be traced precisely, and the accuracy of the approximation drops remarkably. The discontinuous shape under $100 \mathrm{~nm}$ localization precision cannot be represented well by Eq. (1), thereby leading to a significantly larger fitting error than that under $1 \mathrm{~nm}$ localization precision. Given that $k_{\mathrm{B}} T$ is constant at a specific temperature, the flexural rigidity, $\kappa$, can be derived from the slope between $q_{\mathrm{n}}{ }^{4}$ and $L^{4} /$ $\operatorname{var}\left(a_{\mathrm{n}}\right)$ through Eq. (3) [52]. Figure 2C and D show the relationship between $q_{\mathrm{n}}^{4}$ and $L^{4} / \operatorname{var}\left(a_{\mathrm{n}}\right)$ with localization precision of (C) $1 \mathrm{~nm}$ and (D) $100 \mathrm{~nm}$ on a logarithmic graph. When the localization precision is high, $q_{\mathrm{n}}{ }^{4}$ and $L^{4} / \operatorname{var}\left(a_{\mathrm{n}}\right)$ are linearly related regardless of the number of modes (Fig. 2C). However, when the localization precision is low, $q_{\mathrm{n}}{ }^{4}$ and $L^{4} / \operatorname{var}\left(a_{\mathrm{n}}\right)$ maintain a linear relationship only in the low-order mode $(n \leq 4)$
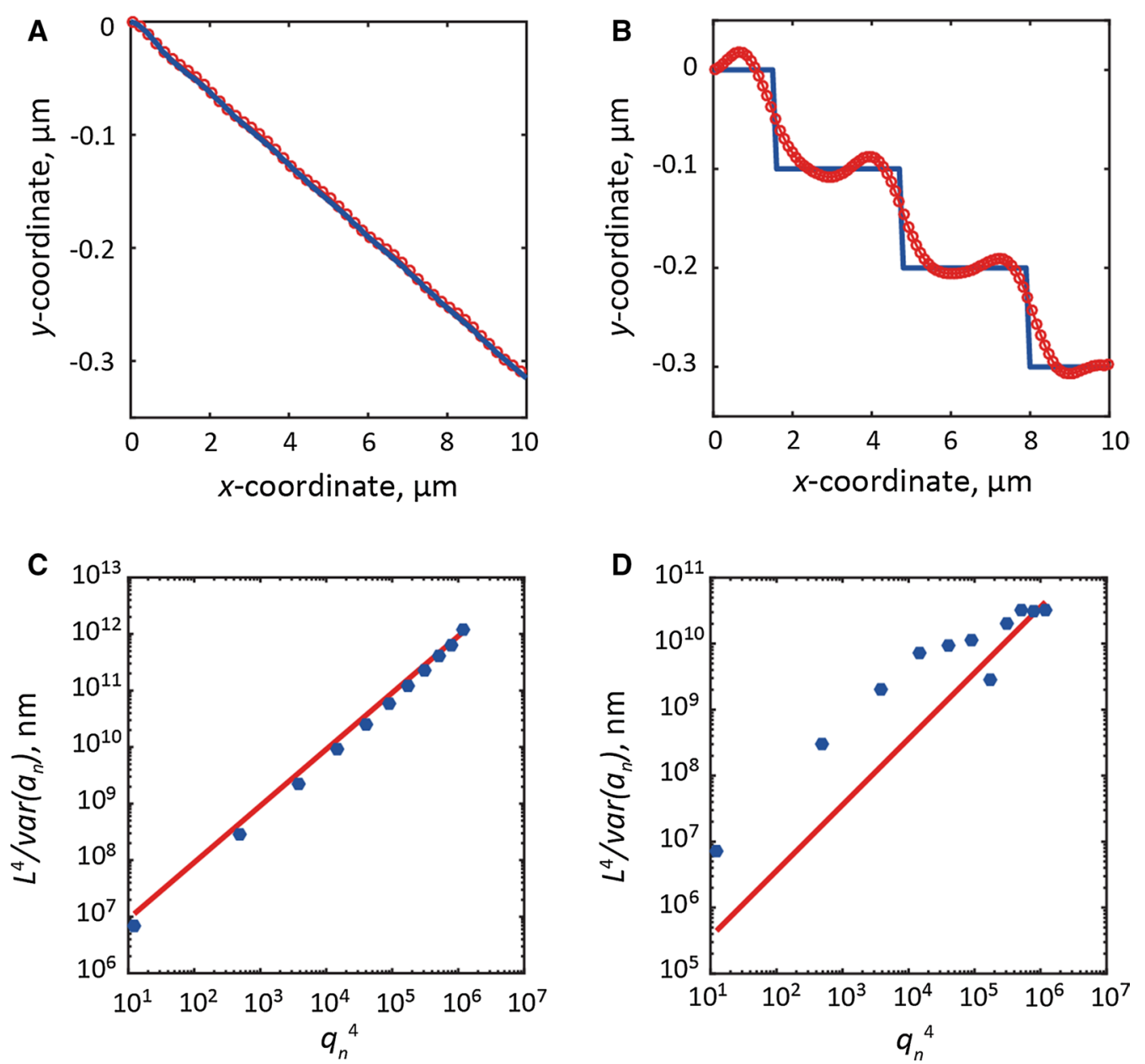

Fig. 2 Measurement process of $k$ for an MT of $L=10 \mu \mathrm{m}$ and $\kappa_{\text {set }}=0.3 \times 10^{-23} \mathrm{~N} \cdot \mathrm{m}^{2}$ with different localization precision. MT shapes (blue lines) and fitted curves (red lines and dots) with localization precision of $\mathbf{A} 1 \mathrm{~nm}$ and $\mathbf{B} 100 \mathrm{~nm}$. Relationship between $q_{n}^{4}$ and $L^{4} / v a r\left(a_{n}\right)$ with localization precision of $\mathbf{C} 1 \mathrm{~nm}$ and $\mathbf{D} 100 \mathrm{~nm}$. Blue dots: measurement results. Red lines: fitted straight lines 
(Fig. 2D). This is because thermal fluctuation cannot be precisely tracked in the higher-order $\left(q_{\mathrm{n}}\right)$ mode, in which the deformation of the shape of MTs is smaller than the localization precision.

\section{Nanometre-level localization precision eliminates the measurement error}

The relative measurement error of $\kappa$ due to the digitization of the shape of MTs was determined from $1-\kappa_{\text {meas }} / \kappa_{\text {set }}$. Figure 3 shows the relative measurement error of flexural rigidity under each condition of $L(5,10$, and $30 \mu \mathrm{m}), \kappa_{\text {set }}\left(0.03,0.3,3 \times 10^{-23} \mathrm{~N} \cdot \mathrm{m}^{2}\right)$, and localization precision of (A) $1 \mathrm{~nm},(B) 10 \mathrm{~nm}$, and (C) $100 \mathrm{~nm}$. The measurement error was found to be affected by the localization precision, MT length, $L$, and the defined flexural rigidity, $\kappa_{\text {set }}$. Lower localization precision, smaller contour lengths, and larger $\kappa_{\text {set }}$ will all result in larger measurement errors. Under low localization precision (10 and $100 \mathrm{~nm}$ ), the flexural rigidity is largely underestimated, especially for short and/or stiff MTs, by overestimating thermal fluctuations.

These results help explain previous discrepancies reported in the literature. For instance, Venier et al. [24] reported a three-fold smaller $\kappa$ than Valdman et al. [35]. Both studies adopted almost the same conditions (tubulin concentration, nucleotide, MT length, and paclitaxel stabilization) but different localization precision for determining MT shape (1 pixel for Venier et al. [24] and 0.1 pixels for Valdman et al. [35]). As indicated in Fig. 3C, the pixel-level localization precision of Venier et al. [24] may have caused the underestimation of $\kappa$. The same can be said for the difference in reported $\kappa$ between Hawkins et al. [21] and Lopez et al. [36]. Although most of the conditions were the same, Hawkins et al. [21] skeletonized the MT image with 1-pixel localization precision and reported a 3.5-fold smaller $\kappa$ than Lopez et al. [36] with 0.1-pixel localization precision.

Our previous studies have shown that MT flexural rigidity is independent of $L$ [10]; however, the current results on simulated filaments indicate that the contour length, $L$, influences the measurement accuracy of flexural rigidity. Venier et al. [24] and Mickey and Howard [17] used similar polymerization conditions and pixellevel localization precision, but different lengths of the MTs in each study. Venier et al. [24] analyzed shorter MTs $(8-15 \mu \mathrm{m})$ than Mickey and Howard [17] (24$68 \mu \mathrm{m})$ and obtained a 2.8 -fold smaller $\kappa$. This is possible because the small deflections of short MTs caused an underestimation of $\kappa$ (Fig. 3C).

The results also indicate that the flexural rigidities of MTs with a large $\kappa$ are more likely to be underestimated, especially at low localization precision. Mickey and Howard [17] reported that $\kappa$ increased about 1.3 times with tau protein bound. Whereas, Felgner et al. [18] used a similar ratio of tau to tubulin and reported that the $\kappa$ increased approximately 2.5 times when measured using optical tweezers. The discrepancy in the increase of $k$ between the two groups is probably caused by the underestimation of $\kappa$ due to the low pixel-level localization precision used by Mickey and Howard [17]. In addition, Cassimeris et al. [19] co-polymerized XMAP215, another type of MAPs, with tubulin and did not find any change in $\kappa$ under pixel-level localization precision. This could be caused due to a very low precision that hinders the detection of changes in $\kappa$ because of the larger measurement error. Thus, it would be interesting to measure the effects of XMAP215 with improved localization precision.

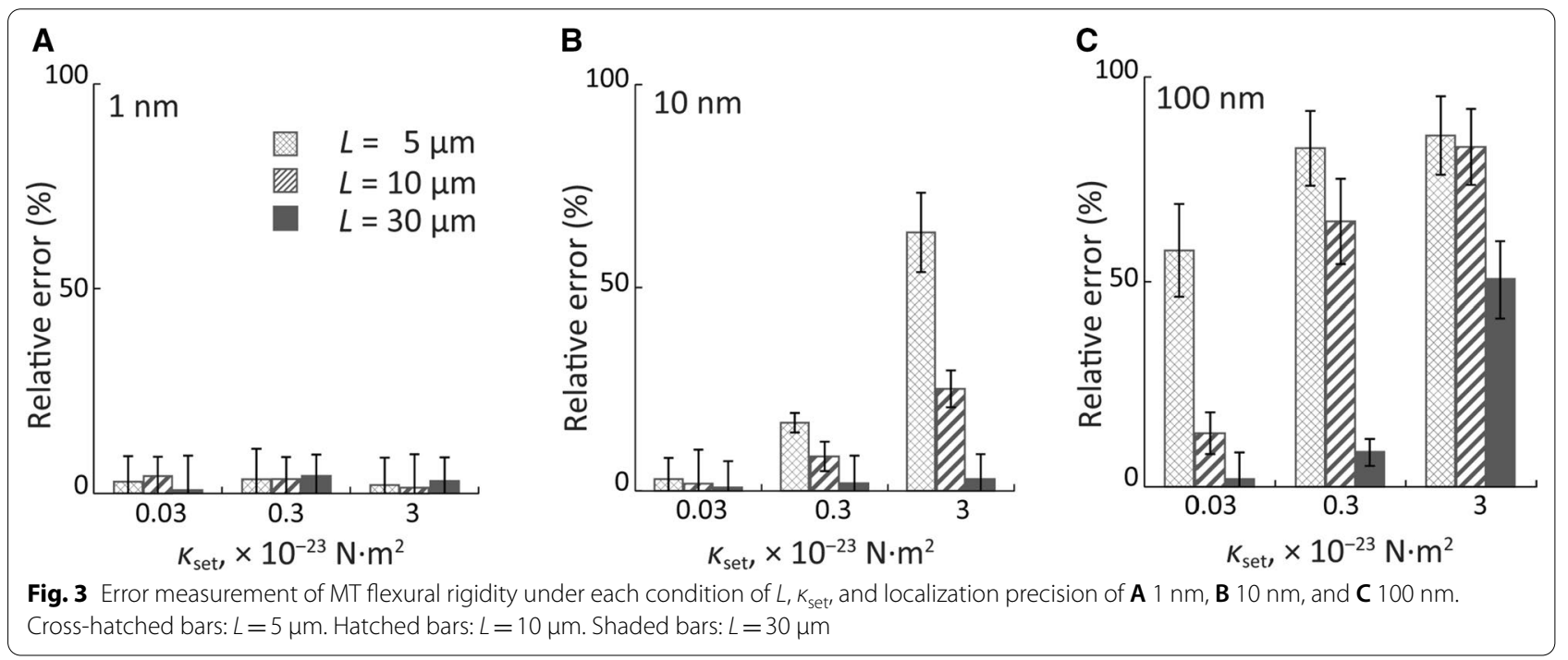


It is noteworthy that, when the localization precision is at $1 \mathrm{~nm}$, the relative measurement error is less than $5 \%$ regardless of the value of $L$ or $\kappa_{\text {set }}$ (Fig. 3A and Additional file 1: Figure S3A). Thus, the measurement error caused by $L$ and $\kappa$ can be significantly reduced by improving the localization precision. Therefore, nanometre-level localization precision is indispensable for the precise and accurate measurement of flexural rigidity for MTs of various lengths and stiffnesses. Further, by improving the localization precision to the nanometre level, it is possible to obtain new findings by reinvestigating the influences of these factors on $\kappa$.

\section{Dependence of MT flexural rigidity on the growth rate} The measured values of flexural rigidity and growth rates of MTs polymerizing with tubulin concentrations of 20-200 $\mu \mathrm{M}$ are summarized in Additional file 1: Table S2. The relationship between the growth rate and flexural rigidity of the MT is shown in Fig. 4B. The growth rate was found to significantly increase with higher tubulin concentrations within the range of $30-150 \mu \mathrm{M}$. The slope of the growth rate was $0.1 \mu \mathrm{m} \mathrm{min}^{-1} \mu \mathrm{M}^{-1}$, which is approximately tenfold faster at $150 \mu \mathrm{M}$ than that at $30 \mu \mathrm{M}$. No significant differences in growth rates were observed between $20 \mu \mathrm{M}$ and $30 \mu \mathrm{M}$ as well as between $150 \mu \mathrm{M}$ and $200 \mu \mathrm{M}$ using the Steel-Dwass test $(p>0.05$, Additional file 1: Table S3). Therefore, the whole variation

\section{A}
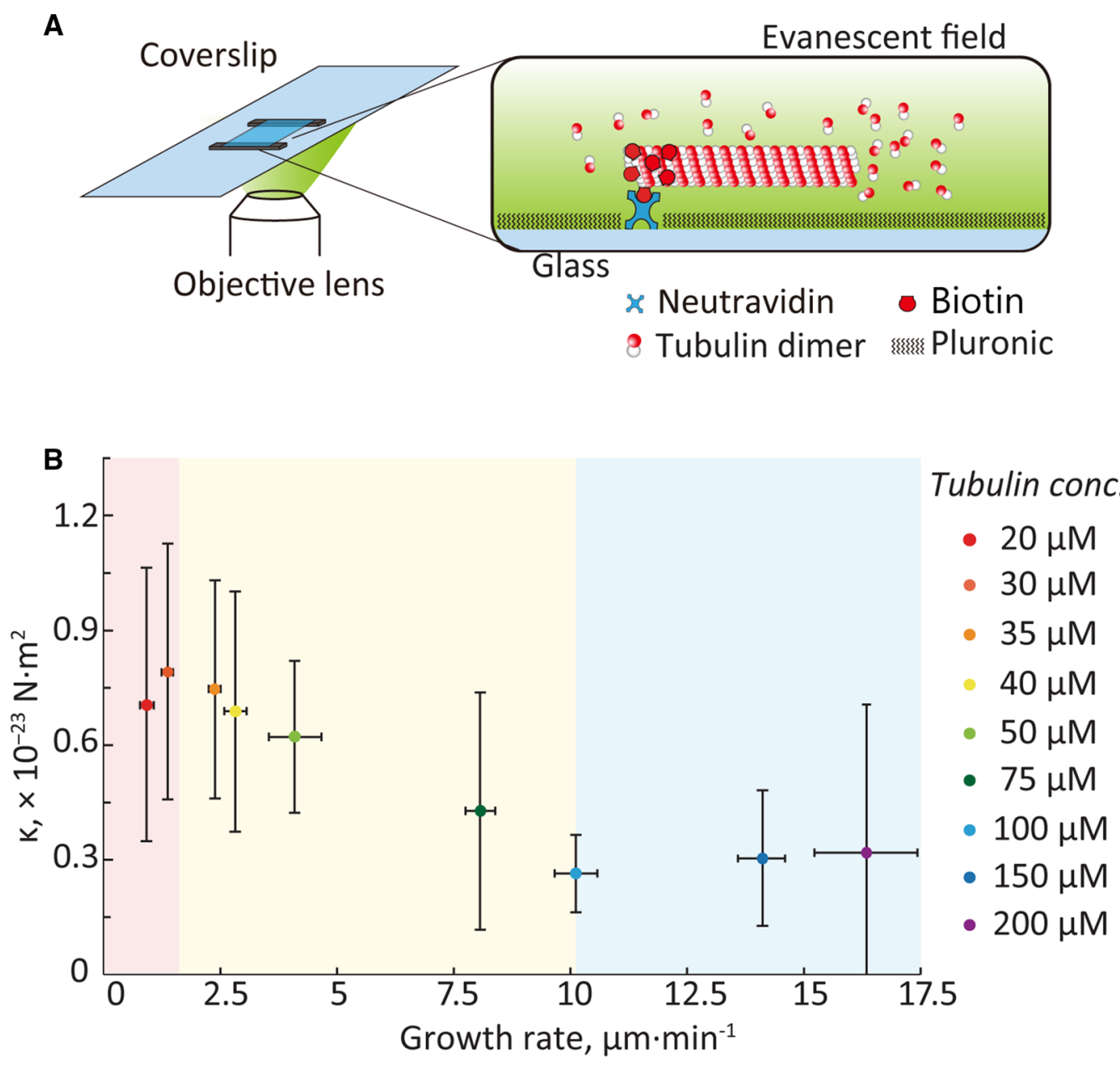

Fig. 4 Relationship between growth rate and flexural rigidity of MTs. A Growth rate measurement of MTs elongating under different tubulin concentrations as observed using TIRF microscopy. The partially biotinylated seed MTs were immobilized onto substrates via biotin-neutravidin bindings. Tubulin protein solution with a specific concentration was introduced into the flow cell and MT elongation process was observed directly. B MT flexural rigidity decreases with an increase in growth rate, which follows a three-state change and is illustrated with red, yellow, and blue background. The corresponding tubulin concentrations of the three stages are $\leq 30 \mu \mathrm{M}, 30-100 \mu \mathrm{M}$, and $>100 \mu \mathrm{M}$, respectively. MTs incubated in tubulin concentrations from 20-200 $\mathrm{\mu M}$ are illustrated by the dots with different colors 

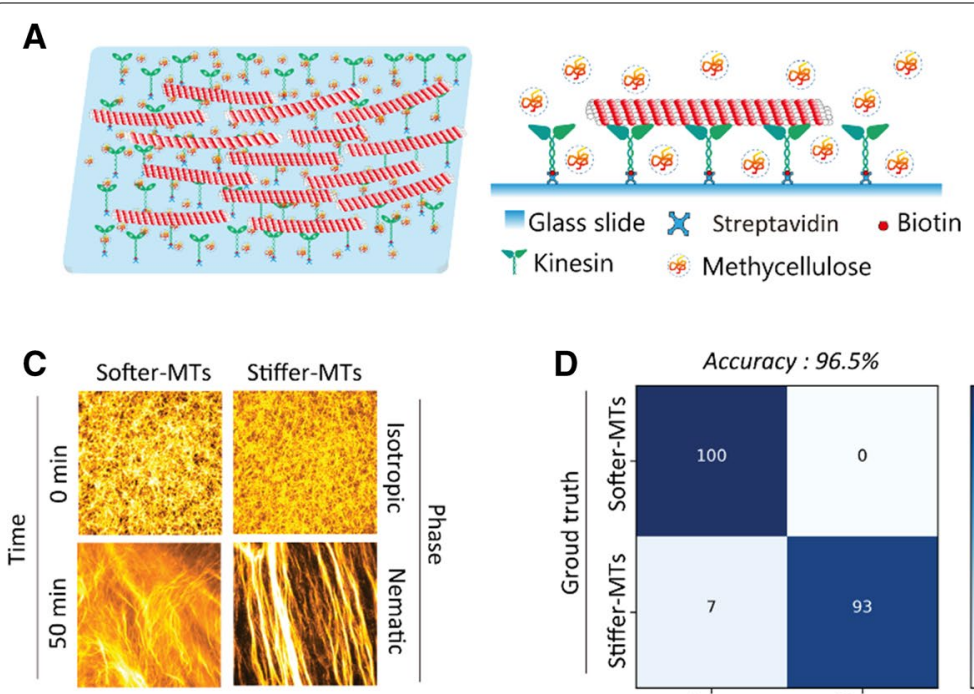

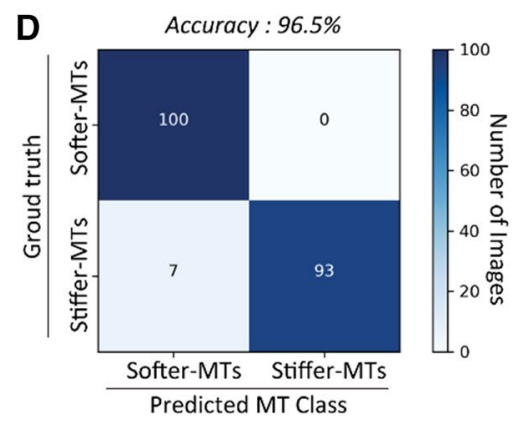

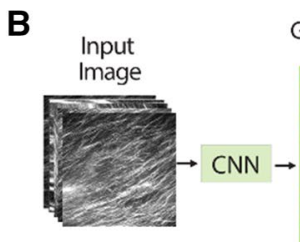
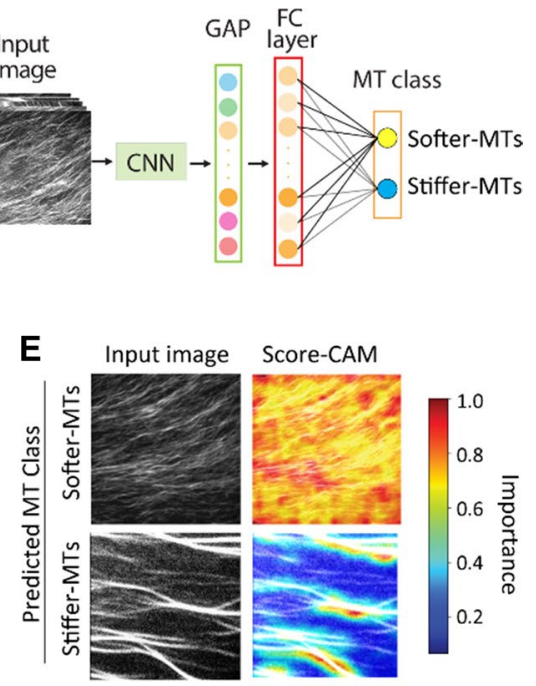

Fig. 5 Distinct patterns formed by MTs with different flexural rigidities. A MT gliding assays conducted within a reconstructed MT-kinesin system in the presence of methylcellulose. B A deep learning CNN model, including global average pooling (GAP) layer, final connected (FC) layer, and MT class output, is constructed to classify the two MTs: softer-MTs and stiffer-MTs. The two groups of MTs are polymerized at different tubulin concentration of $30 \mu \mathrm{M}$ and $100 \mu \mathrm{M}$, which are named as softer-MTs $\left(k=0.27 \times 10^{-23} \mathrm{~N} \mathrm{~m}^{2}\right)$ and stiffer-MTs $\left(k=0.80 \times 10^{-23} \mathrm{~N} \mathrm{~m}^{2}\right)$, respectively. $\mathbf{C}$ From the identical isotropic state, softer-MTs and stiffer-MTs form distinctive patterns at the nematic phase. The addition time of ATP was set as 0 min. Scale bar $=50 \mu \mathrm{m}$. D Confusion matrix based on the trained CNN classifier. Here, 200 MT pattern images are categorized using the classifier. E The classification strategy of CNN classifier is visually explained using Score-CAM

process of growth rate could be divided into three phases according to their statistical differences (Additional file 1: Table S3), with tubulin concentration values of $\leq 30 \mu \mathrm{M}$, $30-100 \mu \mathrm{M}$, and $>100 \mu \mathrm{M}$, which are illustrated by a red, yellow, and blue background, respectively (Fig. 4B).

Meanwhile, flexural rigidity was found to be dependent on the growth rate. When the growth rate was less than $\sim 1.4 \mu \mathrm{m} \mathrm{min}{ }^{-1}$, that is, the tubulin concentration was lower than $30 \mu \mathrm{M}$, the flexural rigidity remained similar to that observed between 20 and $30 \mu \mathrm{M}$ (no statistical significance of MT rigidity at 20 and $30 \mu \mathrm{M}$ was detected, Additional file 1: Table S4). Once the MTs elongate faster than $1.4 \mu \mathrm{m} \mathrm{min}{ }^{-1}$, the flexural rigidity tends to decrease as the growth rate increases. At a growth rate of $\sim 10.1 \mu \mathrm{m} \mathrm{min}{ }^{-1}$ (tubulin concentration higher than $100 \mu \mathrm{M})$, the flexural rigidity becomes independent of the growth rate.

The dependence of the flexural rigidity on the growth rate can be explained by the number of defects generated and repaired during polymerization. As reported by Schaedel et al. [56], faster growth tended to generate lattice defects on MTs, which reduced the MT flexural rigidity. We found that when the tubulin concentration was too low to facilitate fast MT polymerization (20$30 \mu \mathrm{M})$, MTs show high bending stiffness, most likely due to the formation of fewer lattice defects. At a low growth rate, MTs repair their tubulin defects while elongating, thereby resulting in higher flexural rigidity with fewer defects [56].

Fast growth of MTs can result in accumulation of defects that cannot be repaired. Thus, in this regime $(30-100 \mu \mathrm{M})$, higher number of defects in MTs will result in softer filaments. MT rigidity was found to decline monotonically as the growth rate increased in the region (Fig. 4B), which is consistent with the results of Janson and Dogterom $[25,26]$.

As the tubulin concentration during polymerization was increased to over $100 \mu \mathrm{M}$, the growth rate increased and then reached the upper limit value (no significant differences in the growth rate at 150 and $200 \mu \mathrm{M}$ were observed in Additional file 1: Table S3). Correspondingly, no change in rigidity was observed (Fig. 4B, no significant difference of MT rigidities at 100, 150, and $200 \mu \mathrm{M}$ were detected in Additional file 1: Table S4), which indicates that there should be a certain upper limit number of lattice defects accumulating on MTs. Once the quantity of tubulin defects exceeds a certain threshold, fatal structural damages will occur to break MT or cause MT depolymerization. As a result, when MTs grow fast to exceed the upper threshold value $\left(\sim 10.1 \mu \mathrm{m} \mathrm{min}^{-1}\right)$, the number of tubulin defects of the surviving MTs remains constant at the upper limit. Therefore, no additional tubulin defects would be generated, the flexural rigidity of MTs 
keeps at a constant low level with the tubulin concentration higher than $100 \mu \mathrm{M}$.

Results from previous studies display discrepancies in their reported rigidity values owing to the different polymerization speeds. For instance, Valdman et al. [35] and Lopez et al. [36] performed experiments using the same measurement and conditions (localization precision, nucleotide, MT length, and paclitaxel stabilization) except for tubulin concentration (Valdman et al. [35], $20 \mu \mathrm{M}$; Lopez et al. [36], $50 \mu \mathrm{M})$. These publications were from the same group, and therefore, there is a likelihood that the tubulin type was identical. However, the measured $\kappa$ reported by Valdman et al. was approximately 1.4fold larger than that reported by Lopez et al. Although the growth rates were not quantified or reported, it is conceivable that the growth rate observed by Valdman et al. [35] would be smaller than that observed by Lopez et al. [36] because of the difference in the tubulin concentrations. Therefore, we highly recommend future measurements of flexural rigidity to include a measurement of the growth rate for the tubulin polymerization methods used in this study.

Furthermore, our results reveal the relation between MT growth rate and rigidity with a wider range of tubulin concentration $(20-200 \mu \mathrm{M})$ than that in previous studies $(4.5-28 \mu \mathrm{M})[25,26,57]$. For instance, the growth rate and flexural rigidity of MTs polymerizing with a higher tubulin concentration $(>100 \mu \mathrm{M}$, blue area in Fig. 4 B) are $10.1-16.3 \mu \mathrm{m} \mathrm{min}{ }^{-1}$ and $\sim 0.3 \times 10^{-23} \mathrm{~N} \cdot \mathrm{m}^{2}$ (Additional file 1: Table S2), respectively, which corroborate the reported data of MTs in vivo (growth rate of $12-24 \mu \mathrm{m} \mathrm{min}^{-1}$ and flexural rigidity of $0.3-$ $\left.0.4 \times 10^{-23} \mathrm{~N} \cdot \mathrm{m}^{2}\right)[58,59]$. Interestingly, although the reported tubulin concentration in cells $(4-24 \mu \mathrm{M})$ is much lower than that used here $(>100 \mu \mathrm{M})$, growth rates of MTs in cells are still comparable with or even faster than that observed in our results $[60,61]$. The effect of tubulin concentration on the growth of MTs could be compensated by various kinds of MAPs in cells, such as the plus-end tracking proteins, CLASP, CLIP-170, XMAP215, and EB1 [4, 62]. However, the influences of these MAPs on MT rigidity are still unclear. Combined with our results, further experiments on the relationship between growth rate and rigidity in the presence of MAPs will induce an agreement in understanding the factors that influence rigidity in vivo.

Moreover, our results provide a quantitative link between growth rate and flexural rigidity for bare MTs, which suggests a potential method for designing flexural rigidity of MTs in vitro. Flexural rigidity is an important parameter that affects the direction of MT gliding. MTs modified with different flexural rigidities have been widely applied in nano- and bioengineering applications, including molecular shuttles, active self-organization, and collective motion [8-10]. Although the flexural rigidity of MTs can be modified by various MAPs, nucleotides, and MT-stabilizing reagents [16-21, 25-27, 36], they are also known to influence the molecular structure and biophysical functions of MTs [4, 36, 62, 63]. For instance, Janson and Dogterom $[25,26]$ polymerized MTs with/without oxygen-scavenging system (4 mM dithiothreitol, $0.2 \mathrm{mg} \mathrm{ml}^{-1}$ catalase, $0.4 \mathrm{mg} \mathrm{ml}^{-1}$ glucose oxidase, and $50 \mathrm{mM}$ glucose) and found the oxygen-scavenger may promote the polymerization speed of MTs from 1.5 to $2.72 \mu \mathrm{m} \mathrm{min}{ }^{-1}$ and soften MTs from 2.73 to $1.74 \times 10^{-23} \mathrm{~N} \cdot \mathrm{m}^{2}$ (Additional file 1: Table S1). Their studies suggested that regulating growth rate could become a potential method to control MT rigidity, however, no specific implementable scheme to realize this idea so far. Here, the newfound relationship of growth rate and MT rigidity controlled by tubulin concentration addressed this issue. Compared with these conventional modification methods [16-21, 25-27, 36], our growth rate-dependent method is controlled solely by the concentration of tubulin. Therefore, the method not only simplifies the preparation of MTs, but also avoids uncertain influences on MTs, which will widen the applicability of the flexural rigidity for engineering applications.

\section{Growth rate-dependent flexural rigidity influences the collective motion in the MT motility assay}

Two groups of MTs were used to perform the MT gliding assays. Softer-MTs were polymerized at $100 \mu \mathrm{M}$ tubulin concentration with the growth rate of $10.10 \pm 0.46 \mu \mathrm{m} \mathrm{min}^{-1}$ and $\kappa$ of $0.27 \pm 0.10 \times 10^{-23} \mathrm{~N} \cdot \mathrm{m}^{2}$, whereas stiffer-MTs were polymerized at $30 \mu \mathrm{M}$ tubulin concentration with the growth rate of $1.37 \pm 0.07 \mu \mathrm{m} \mathrm{min}^{-1}$ and $\kappa$ of $0.80 \pm 0.34 \times 10^{-23} \mathrm{~N} \cdot \mathrm{m}^{2}$ (Fig. 4B and Additional file 1: Table S2).

Distinct patterns of collective motion emerged in the two groups of MTs during the gliding assays. Initially, MTs in both groups were uniformly distributed with random orientations, which could be treated as an isotropic phase (0 min, Fig. $5 \mathrm{C})$. Next, the gliding MTs continually collided with their neighboring MTs in the presence of a depletion force induced by methylcellulose. The gliding directions and orientations of the MTs were tuned by frequent collisions. The collective motion gradually arose in both groups and formed stable nematic patterns after $50 \mathrm{~min}$. Softer MTs tended to form local steam, whereas stiffer MTs formed stable bundles (50 min, Fig. 5C).

MT patterns formed (at $50 \mathrm{~min}$ ) by the two MT groups were categorized into two classes, softer-MTs and stifferMTs, using the trained deep CNN classifier. The 200 MT pattern images were categorized using the trained CNN classifier with an accuracy of $96.5 \%$ (100\% accuracy for 
softer-MTs and 93\% accuracy for stiffer-MTs) (Fig. 5D). The types of MTs could be predicted and sorted into their corresponding classes according to their patterns, which suggested certain general differences in patterns between the two groups.

The differences in patterns between the two groups were revealed by analyzing the classification strategy of the CNN classifier. Score-CAM was introduced to visually explain how the classifier categorized the MT patterns into the two groups (Fig. 5E) [55]. The more important the area or feature in the image for making the final categorization decision by the classifier, the higher the score gained and the stronger the responses in the CAM. For the images sorted into softer-MT groups, a uniformly high activation responded and overlaid the entire image area, which indicated that a large-scale local stream with a relatively even distribution was detected (Fig. 5E). In contrast, for the images sorted into the stiffer-MT group, only the bundle area was highly activated in the heatmap, which indicated that a distinguished uneven structure (MT bundle) was identified (Fig. 5E). The differences in the ScoreCAM patterns between the two groups were in accordance with our observed results.

The collective motion of MTs has been proposed to emerge due to a depletion force between filaments, which reduces filament crossover, promotes alignment, and leads to a transition of filaments from an active, isotropic state to locally aligned polar patterns [64]. According to the Vicsek model, identical objects, including particles or filaments moving at a constant speed, tend to interact locally by aligning with neighbors [65]. A similar opinion was proposed that self-propelled objects, such as gilding MTs, show progressively larger probabilities to align with their neighbors [66]. Recently, an important supplement to these hypotheses stated that MTs align and are actively transferred from their original bundle to another antialigned bundle [67]. All the theories indicated that alignment is a critical step in the formation of collective motion, and the formation of MT bundles is a dynamic process. As an apparent and key factor affecting the gliding and alignment behavior of the filaments, MT flexural rigidity should directly influence the phase transition process of collective motion.

The two groups of MTs enable us to infer that MTs with different $\kappa$ perform differently on the occasions of collision, alignment, and diffusive separation. Compared with the stiffer-MTs, the softer-MTs are more flexible in changing their gliding directions. Although the bundles of softer MTs were formed by the depletion force, the MTs inside the bundle could be easily dispersed in the next collision or disturbed by another anti-aligned bundle. In contrast, the bundles formed by stiffer-MTs are long-standing and tend to merge with neighboring bundles, which results in a relatively large bundle. Moreover, the performance differentials of defined softer MTs and stiffer-MTs demonstrated that the method for designing flexural rigidity is feasible.

\section{Conclusions}

In summary, an accurate method of measuring MT flexural rigidity was developed by improving the localization precision up to nanometer scale. Based on this improved methodology, we further discovered that flexural rigidity is directly affected by growth rate and goes through three phases among a wide range of tubulin concentrations $(20-200 \mu \mathrm{M})$, which revealed a new relationship between dynamics and mechanics of MTs and deepened the understanding of the regulatory mechanism of MT flexural rigidity in vivo. These findings not only offer reasonable explanations for long-standing discrepancies in previously measured flexural rigidities, but also provide a convenient method for the production of MTs with predefined stiffness.

Consequently, as shown with the example of MT collective motion, the proposed method would promote the development of potential nanobiotechnology applications by eliminating methodological inconsistencies among assays and providing a controllable experimental environment. Additionally, owing to the simplicity and ease of manipulation, the method for designing flexural rigidity of MTs by altering tubulin concentrations will be a useful quantitative tool to investigate the biological functions of MT rigidity or study the influences of MT flexural rigidity on their routine behaviors in vitro, including gliding motility assays, collective motion, and other biological activities.

Besides tubulin concentration, the regulations of the dynamics and mechanics of MTs in vivo involve many other factors, including MAPs and post-translational modifications. Our results revealed a new quantitative relation between the growth rate and flexural rigidity of MTs by solely modifying the tubulin concentration. It provides comprehensive and comparable data of growth rate and MT rigidity with that in vivo and can be a datum reference for investigating the regulation mechanism of MT mechanics and the functions of specific MAP in the future. 


\section{Supplementary Information}

The online version contains supplementary material available at https://doi. org/10.1186/s12951-021-00960-y.

Additional file 1: Figure S1. The fabrication process of Au stripepatterned substrate. Figure $\mathbf{S 2}$. Measurement process of MT flexural rigidity. Figure $\mathbf{S} 3$. Summary of mean values of $K_{\text {meas }} / K_{\text {set. }}$. Figure $\mathbf{S} 4$. Measurement process of MT growth rate. Figure $\mathbf{S 5}$. The training process and performance evaluation of the CNN classifier. Table S1. Summary of reported flexural rigidity for MTs. Table S2. Summary of the flexural rigidity and growth rate of MTs polymerized using 20-200 $\mu \mathrm{M}$ tubulin. Table S3. $P$ values of the Steel-Dwass test for MT growth rate at different tubulin concentrations. Table S4. $P$ values of the Steel-Dwass test for MT flexural rigidity at different tubulin concentrations.

Additional file 2: Movie S1. The fluctuation of MT polymerizing under $20 \mu \mathrm{M}$ tubulin concentration.

Additional file 3: Movie S2. The fluctuation of MT polymerizing under $30 \mu \mathrm{M}$ tubulin concentration.

Additional file 4: Movie S3. The fluctuation of MT polymerizing under $50 \mu \mathrm{M}$ tubulin concentration.

Additional file 5: Movie S4. The fluctuation of MT polymerizing under $100 \mu \mathrm{M}$ tubulin concentration.

\section{Acknowledgements}

We thank Jennifer L. Ross and Taviare L. Hawkins for sharing expertise and $M A T L A B$ codes on evaluating the mechanics of microtubules.

\section{Authors' contributions}

$\mathrm{HZ}, \mathrm{NI}$, and $\mathrm{RY}$ designed the experiments; $\mathrm{HZ}$ and $\mathrm{NI}$ performed the experiments and analyzed the data; all authors discussed, interpreted the results, and wrote the paper. All authors read and approved the final manuscript.

\section{Funding}

This study was partially supported by the Japan Society for the Promotion of Science (JSPS) KAKENHI (Grant Numbers 17H03206, 20H00330); JKA and its promotion funds from KEIRIN RACE; Tateisi Science and Technology Foundation; Kyoto University Nano Technology Hub in "Nanotechnology Platform Project" sponsored by MEXT, Japan. H.Z. was supported by MEXT scholarship (Grant Number 171560). N.I. was supported by a Grant-in-Aid for JSPS Research Fellow (Grant Number 262439).

\section{Declarations}

\section{Consent for publication}

All authors agreed to submit this manuscript.

\section{Competing interests}

There are no conflicts to declare.

Received: 28 June 2021 Accepted: 11 July 2021

Published online: 19 July 2021

\section{References}

1. Mücke N, Kreplak L, Kirmse R, Wedig T, Herrmann H, Aebi U. Assessing the flexibility of intermediate filaments by atomic force microscopy. J Mol Biol. 2004;335:1241-50

2. Pallavicini $C$, Levi $V$, Wetzler $D E$, Angiolini JF, Benseñor L, Despósito MA. Lateral motion and bending of microtubules studied with a new single-filament tracking routine in living cells. Biophys J. 2014;106:2625-35.

3. Fletcher DA, Mullins RD. Cell mechanics and the cytoskeleton. Nature. 2010:463:485-92.

4. Hawkins T, Mirigian M, Selcuk Yasar M, Ross JL. Mechanics of microtubules. J Biomech. 2010:43:23-30.
5. Sumino Y, Nagai KH, Shitaka Y, Tanaka D, Yoshikawa K, Chaté H. Largescale vortex lattice emerging from collectively moving microtubules. Nature. 2012:483:448-52.

6. Dennis JR, Howard J, Vogel V. Molecular shuttles: directed motion of microtubules along nanoscale kinesin tracks. Nanotechnology. 1999;10:232-6

7. Hess H, Ross JL. Non-equilibrium assembly of microtubules: from molecules to autonomous chemical robots. Chem Soc Rev. 2017;46:5570-87.

8. Wada S, Rashedul Kabir AM, Ito M, Inoue D, Sada K, Kakugo A. Effect of length and rigidity of microtubules on the size of ring-shaped assemblies obtained through active self-organization. Soft Matter. 2015;11:1151-7.

9. Farhadi L, Fermino Do Rosario C, Debold EP, Baskaran A, Ross JL. Active self-organization of actin-microtubule composite self-propelled rods. Front Phys. 2018;6:75.

10. Isozaki N, Shintaku H, Kotera H, Hawkins TL, Ross JL, Yokokawa R. Control of molecular shuttles by designing electrical and mechanical properties of microtubules. Sci Robot. 2017;2:aan4882.

11. Kaneko T, Furuta K, Oiwa K, Shintaku H, Kotera H, Yokokawa R. Different motilities of microtubules driven by kinesin-1 and kinesin-14 motors patterned on nanopillars. Sci Adv. 2020;6:eaax7413.

12. Van den Heuvel MGL, Bolhuis S, Dekker C. Persistence length measurements from stochastic single-microtubule trajectories. Nano Lett. 2007;7:3138-44.

13. Bouxsein NF, Bachand GD. Single filament behavior of microtubules in the presence of added divalent counterions. Biomacromol. 2014;15:3696-705.

14. Van den Heuvel MGL, De Graaff MP, Dekker C. Microtubule curvatures under perpendicular electric forces reveal a low persistence length. Proc Natl Acad Sci U S A. 2008;105:7941-6.

15. Mizushima-Sugano J, Maeda T, Miki-Noumura T. Flexural rigidity of singlet microtubules estimated from statistical analysis of their contour lengths and end-to-end distances. Biochim Biophys Acta BBA. 1983;755:257-62.

16. Dye RB, Fink SP, Williams RC. Taxol-induced flexibility of microtubules and its reversal by MAP-2 and Tau. J Biol Chem. 1993;268:6847-50.

17. Mickey B, Howard J. Rigidity of microtubules is increased by stabilizing agents. J Cell Biol. 1995;130:909-17.

18. Felgner $\mathrm{H}$, Frank R, Biernat J, Mandelkow E-M, Mandelkow E, Ludin B. Domains of neuronal microtubule-associated proteins and flexural rigidity of microtubules. J Cell Biol. 1997;138:1067-75

19. Cassimeris L, Gard D, Tran PT, Erickson HP. XMAP215 is a long thin molecule that does not increase microtubule stiffness. J Cell Sci. 2001:114:3025-33.

20. Kawaguchi K, Yamaguchi A. Temperature dependence rigidity of nontaxol stabilized single microtubules. Biochem Biophys Res Commun. 2010;402:66-9.

21. Hawkins TL, Sept D, Mogessie B, Straube A, Ross JL. Mechanical properties of doubly stabilized microtubule filaments. Biophys J. 2013;104:1517-28.

22. Gittes F. Flexural rigidity of microtubules and actin filaments measured from thermal fluctuations in shape. J Cell Biol. 1993:120:923-34.

23. Felgner $H$, Frank $R$, Schliwa M. Flexural rigidity of microtubules measured with the use of optical tweezers. J Cell Sci. 1996:109·509-16.

24. Venier P, Maggs AC, Carlier MF, Pantaloni D. Analysis of microtubule rigidity using hydrodynamic flow and thermal fluctuations. J Biol Chem. 1994;269:13353-60.

25. Janson ME, Dogterom M. Scaling of Microtubule Force-Velocity Curves Obtained at Different Tubulin Concentrations. Phys Rev Lett. 2004;92:248101.

26. Janson ME, Dogterom M. A bending mode analysis for growing microtubules: evidence for a velocity-dependent rigidity. Biophys J. 2004:87:2723-36.

27. Dogterom M, Yurke B. Measurement of the force-velocity relation for growing microtubules. Science. 1997:278:856-60.

28. Kikumoto $M$, Kurachi $M$, Tosa $V$, Tashiro $H$. Flexural rigidity of individual microtubules measured by a buckling force with optical traps. Biophys J. 2006;90:1687-96

29. Kis A, Kasas S, Babić B, Kulik AJ, Benoît W, Briggs GAD. Nanomechanics of microtubules. Phys Rev Lett. 2002;89:248101.

30. Brangwynne CP, Koenderink GH, Barry E, Dogic Z, MacKintosh FC, Weitz DA. Bending dynamics of fluctuating biopolymers probed by automated high-resolution filament tracking. Biophys J. 2007;93:346-59. 
31. Portran D, Zoccoler M, Gaillard J, Stoppin-Mellet V, Neumann E, Arnal I. MAP65/Ase1 promote microtubule flexibility. Mol Biol Cell. 2013;24:1964-73.

32. Pampaloni F, Lattanzi G, Jonáš A, Surrey T, Frey E, Florin E-L. Thermal fluc tuations of grafted microtubules provide evidence of a length-dependent persistence length. Proc Natl Acad Sci U S A. 2006;103:10248-53.

33. Taute KM, Pampaloni F, Frey E, Florin E-L. Microtubule dynamics depart from the Wormlike Chain Model. Phys Rev Lett. 2008;100:028102.

34. Vale RD, Coppin CM, Malik F, Kull FJ, Milligan RA. Tubulin GTP hydrolysis influences the structure, mechanical properties, and kinesin-driven transport of microtubules. J Biol Chem. 1994;269:23769-75.

35. Valdman D, Atzberger PJ, Yu D, Kuei S, Valentine MT. Spectral Analysis methods for the robust measurement of the flexural rigidity of biopolymers. Biophys J. 2012;102:1144-53.

36. Lopez BJ, Valentine MT. Mechanical effects of EB1 on microtubules depend on GTP hydrolysis state and presence of paclitaxel: effects of EB1 on microtubule mechanics. Cytoskeleton. 2014;71:530-41.

37. Peck A, Sargin ME, LaPointe NE, Rose K, Manjunath BS, Feinstein SC. Tau isoform-specific modulation of kinesin-driven microtubule gliding rates and trajectories as determined with tau-stabilized microtubules. Cytoskeleton. 2011;68:44-55.

38. Van Mameren J, Vermeulen KC, Gittes F, Schmidt CF. Leveraging single protein polymers to measure flexural rigidity. J Phys Chem B. 2009:113:3837-44.

39. Schaedel L, John K, Gaillard J, Nachury MV, Blanchoin L, Théry M. Microtubules self-repair in response to mechanical stress. Nat Mater 2015:14:1156-63.

40. Janke C, Magiera MM. The tubulin code and its role in controlling microtubule properties and functions. Nat Rev Mol Cell Biol. 2020;21:307-26.

41. Zanic M, Widlund PO, Hyman AA, Howard J. Synergy between XMAP215 and EB1 increases microtubule growth rates to physiological levels. Nat Cell Biol. 2013;15:688.

42. Kerssemakers JWJ, Munteanu EL, Laan L, Noetzel TL, Janson ME, Dogterom M. Assembly dynamics of microtubules at molecular resolution. Nature. 2006:442:709.

43. Kinoshita K, Arnal I, Desai A, Drechsel DN, Hyman AA. Reconstitution of physiological microtubule dynamics using purified components. Science. 2001;294:1340-3

44. Vasquez RJ, Howell B, Yvon AM, Wadsworth P, Cassimeris L. Nanomolar concentrations of nocodazole alter microtubule dynamic instability in vivo and in vitro. Mol Biol Cell. 1997;8:973-85.

45. Komarova Y, Groot COD, Grigoriev I, Gouveia SM, Munteanu EL, Schober JM. Mammalian end binding proteins control persistent microtubule growth. J Cell Biol. 2009;184:691-706.

46. Jordan MA, Kamath K, Manna T, Okouneva T, Miller HP, Davis C. The primary antimitotic mechanism of action of the synthetic halichondrin E7389 is suppression of microtubule growth. Mol Cancer Ther. 2005;4:1086-95.

47. Pedigo S, Williams RC. Concentration dependence of variability in growth rates of microtubules. Biophys J. 2002;83:1809-19.

48. Drechsel DN, Hyman AA, Cobb MH, Kirschner MW. Modulation of the dynamic instability of tubulin assembly by the microtubule-associated protein tau. Mol Biol Cell. 1992:3:1141-54.

49. Castoldi M, Popov AV. Purification of brain tubulin through two cycles of polymerization-depolymerization in a high-molarity buffer. Protein Expr Purif. 2003;32:83-8.

50. Yokokawa R, Tarhan MC, Kon T, Fujita H. Simultaneous and bidirectional transport of kinesin-coated microspheres and dynein-coated microspheres on polarity-oriented microtubules. Biotechnol Bioeng. 2008:101:1-8
51. Wiggins CH, Riveline D, Ott A, Goldstein RE. Trapping and wiggling: elastohydrodynamics of driven microfilaments. Biophys J. 1998;74:1043-60.

52. Hawkins TL, Mirigian M, Li J, Yasar MS, Sackett DL, Sept D. Perturbations in microtubule mechanics from tubulin preparation. Cell Mole Bioeng. 2012;5:227-38.

53. Ruhnow F, Zwicker D, Diez S. Tracking single particles and elongated filaments with nanometer precision. Biophys J. 2011;100:2820-8.

54. He K, Zhang X, Ren S, Sun J. Deep residual learning for image recognition. In Proceedings of the 2016 IEEE Conference on Computer Vision and Pattern Recognition (CVPR); 2016. pp. 770-8.

55. Wang H, Wang Z, Du M, Yang F, Zhang Z, Ding S. Score-CAM: Scoreweighted visual explanations for convolutional neural networks. In Proceedings of the 2020 IEEE/CVF Conference on Computer Vision and Pattern Recognition Workshops (CVPRW); 2020. pp. 111-9.

56. Schaedel L, Triclin S, Chrétien D, Abrieu A, Aumeier C, Gaillard J. Lattice defects induce microtubule self-renewal. Nat Phys. 2019;15:830-8.

57. Chretien D. Structure of growing microtubule ends: two-dimensional sheets close into tubes at variable rates. J Cell Biol. 1995;129:1311-28.

58. Zwetsloot AJ, Tut G, Straube A. Measuring microtubule dynamics. Essays Biochem. 2018:62:725-35.

59. Battle C, Ott CM, Burnette DT, Lippincott-Schwartz J, Schmidt CF. IntracelIular and extracellular forces drive primary cilia movement. Proc Natl Acad Sci U S A. 2015;112:1410-5.

60. Gard DL, Kirschner MW. Microtubule assembly in cytoplasmic extracts of Xenopus oocytes and eggs. J Cell Biol. 1987;105:2191-201.

61. Loiodice I, Janson ME, Tavormina P, Schaub S, Bhatt D, Cochran R. Quantifying tubulin concentration and microtubule number throughout the fission yeast cell cycle. Biomolecules. 2019;9:86.

62. Akhmanova A, Steinmetz MO. Tracking the ends: a dynamic protein network controls the fate of microtubule tips. Nat Rev Mol Cell Biol. 2008;9:309-22.

63. Zhang R, LaFrance B, Nogales E. Separating the effects of nucleotide and EB binding on microtubule structure. Proc Natl Acad Sci U S A. 2018;115:E6191-200.

64. Inoue D, Mahmot B, Rashedul Kabir AM, Ishrat Farhana T, Tokuraku K, Sada $\mathrm{K}$. Depletion force induced collective motion of microtubules driven by kinesin. Nanoscale. 2015;7:18054-61.

65. Vicsek T, Czirók A, Ben-Jacob E, Cohen I, Shochet O. Novel type of phase transition in a system of self-driven particles. Phys Rev Lett. 1995;75:1226-9.

66. Peruani F, Klauss T, Deutsch A, Voss-Boehme A. Traffic jams, gliders, and bands in the quest for collective motion of self-propelled particles. Phys Rev Lett. 2011;106:128101.

67. Ravichandran A, Duman Ö, Hoore M, Saggiorato G, Vliegenthart GA Auth T. Chronology of motor-mediated microtubule streaming. eLife. 2019;8:e39694.

\section{Publisher's Note}

Springer Nature remains neutral with regard to jurisdictional claims in published maps and institutional affiliations.

Ready to submit your research? Choose BMC and benefit from:

- fast, convenient online submission

- thorough peer review by experienced researchers in your field

- rapid publication on acceptance

- support for research data, including large and complex data types

- gold Open Access which fosters wider collaboration and increased citations

- maximum visibility for your research: over 100M website views per year

At BMC, research is always in progress.

Learn more biomedcentral.com/submissions 